\title{
Transferência de conhecimento: a perspectiva empresarial
}

Ernani Carvalho da Costa Neto

Pós-graduação em Marketing (ESPM) e MBA (INSEAD). Professor e coordenador do Núcleo de Agronegócios da ESPM Sul

ernani.neto@espm.br

\section{Marcelo Gattermann Perin}

Doutor em Administração pela Universidade Federal do Rio Grande do Sul . Professor titular nos cursos de Mestrado e Doutorado do Programa de Pós-Graduação em Administração e coordenador de pesquisa da Escola de Negócios na Pontifícia Universidade Católica do Rio Grande do Sul. ernani.neto@espm.br

\section{Gabriela Cardozo Ferreira}

Doutora em Administração pela Universidade Federal do Rio Grande do Sul. Professora titular da Pontifícia Universidade Católica do Rio Grande do Sul e pesquisadora na área de Estratégia, Competitividade, Inovação e Empreendedorismo.

gcferreira@pucrs.br

\section{Editor Científico: José Edson Lara Organização Comitê Científico \\ Double Blind Review pelo SER/OJS \\ Recebido em 30.10.2018 \\ Aprovado em 12.03.2019}

\section{cc) (7) (8)}

Este trabalho foi licenciado com uma Licença Creative Commons - Atribuição - Não Comercial 3.0 Brasil 


\title{
Resumo
}

Competitividade e diminuição dos ciclos tecnológicos têm conduzido as empresas a buscar diversas fontes de inovação, com destaque para a interação entre universidade e empresa, por meio da transferência de conhecimento. Entretanto, as diferenças de cultura e estrutura organizacionais de universidades e de empresas representam desafios à efetividade do processo de transferência. Assim, esse artigo explora dimensões relevantes no processo de interação universidade e empresa, tendo como perspectiva a visão de empresas inovadoras do ambiente econômico Brasileiro. Foram entrevistados 14 executivos de empresas com processos de interação com universidades, e identificadas cinco dimensões na visão das empresas: as motivações para se engajar nas colaborações, as atividades de transferência realizadas nas interações, os fatores intervenientes, facilitadores e barreiras às transferências, assim como os resultados de inovação esperados. Foram também avaliados os principais indicadores componentes de cada uma destas dimensões, considerada a realidade de empresas Brasileiras.

Palavras-chave: Interação universidade-empresa; Transferência de conhecimento; Inovação.

\section{Knowledge transferring: the business perspective}

\begin{abstract}
The increasing competitiveness and reduction of technological cycles have led companies to search for different sources of innovation, with emphasis on the interaction between university and industry, through the transfer of knowledge and technology. However, differences in organizational culture and structure of universities and industries represent challenges to the effectiveness of the transfer process. Therefore, this article explores the relevant dimensions in the interaction process between university and industry. It presents the perspective of innovative companies of the Brazilian economic environment. We interviewed 14 executives from businesses with universities' interaction. Five dimensions were identified in the companies' view: the motivations to engage in the collaborations, the transfer activities carried out in the interactions, the intervening factors, transfer facilitators and barriers, as well as the innovation results expected from interaction with universities. Dimensions indicators were also identified, considered the reality of Brazilian companies.
\end{abstract}

Key words: University-company interaction; Transfer of knowledge; Innovation.

\section{Transferencia de conocimiento: la perspectiva empresarial}

\section{Resúmen}

Competitividad y disminución de los ciclos tecnológicos han conducido a las empresas a buscar diversas fuentes de innovación, con destaque para la interacción con universidades, a través de la transferencia de conocimiento. Pero, las diferencias de cultura y estructura organizacional de universidades y de empresas representan desafíos a la efectividad de la transferencia. Así, este artículo explora dimensiones relevantes del proceso de interacción universidad y empresa, 
teniendo como perspectiva la visión de empresas innovadoras del ambiente económico brasileño. Se entrevistó a 14 ejecutivos de empresas con procesos de interacción con universidades. Fueran identificadas cinco dimensiones en la visión de las empresas: motivaciones para involucrarse en las colaboraciones, actividades de transferencia realizadas en las interacciones, factores intervinientes, facilitadores y barreras a las transferencias, así como resultados de innovación esperados. También se evaluaron los principales indicadores componentes de cada una de estas dimensiones, considerada la realidad de empresas brasileñas.

Palabras clave: Interacción universidad-empresa; Transferencia de conocimiento; Innovación.

\section{Introdução}

A competição entre empresas está na essência da inovação como ferramenta para sustentar seus diferenciais competitivos (Kahn, 2018). Com a busca por inovação pelas empresas, ganham relevância as alianças e as cooperações em pesquisa e desenvolvimento (Belderbos, Carree, \& Lokshin, 2004). Dentre as formas de buscar inovação, as empresas estabelecem relações com as universidades, que desempenham papel importante na inovação empresarial (de Wit-de Vries et al., 2018). A transferência de conhecimento (TC) a partir de interações com universidades torna-se estratégica para as empresas inovadoras e o êxito deste processo contribui significativamente para ambas instituições (Perkmann, King, \& Pavelin, 2011). Entretanto, a realidade observada em estudos sobre a temática, demonstra uma série de dificuldades na colaboração universidade-empresa (de Wit-de Vries et al., 2018).

A orientação da universidade para a ciência pura e para o longo prazo diverge da orientação comercial da empresa, causando a falta de entendimento comum em relação às expectativas e à dinâmica de trabalho (Bruneel, D'este, \& Salter, 2010). A interação traz adicionalmente desafio ligadas às transações envolvendo os direitos sobre as inovações e deveres das partes envolvidas (Hughes \& Kitson, 2013;).

No Brasil, além de dificuldades similares à realidade internacional, encontra-se um ambiente cujas políticas industriais e de inovação estão, muitas vezes, desarticuladas (Freitas et al., 2013). Tal característica do ambiente causa o distanciamento entre os investimentos em ciência, tecnologia e inovação e a demanda por inovação nas empresas (Closs \& Ferreira, 2012). Infelizmente, as pesquisas sobre o tema abordam majoritariamente economias desenvolvidas, nas quais predominam ambientes favoráveis à inovação, seja pela colaboração institucional do governo, ou por cenários econômicos, sociais e políticos estáveis (Frank, 2016). Por outro lado, 
a literatura apresenta uma ênfase desproporcional ao motivos e benefícios do ponto da universidade no processo (Ryan et al., 2018)

Desta forma, o presente estudo teve como propósito analisar as dimensões relevantes no processo de TC entre universidades e empresas, porém sob a perspectiva empresarial, além de considerar o contexto específico do Brasil.

\section{Interação universidade-empresa na visão empresarial}

O de conceito de TC adotado por este estudo, ocorre no ambiente do processo de interação entre universidade e empresa, conforme sintetizado em modelo proposto por Ankrah e Al-Tabbaa (2015). Tal modelo teórico envolve uma fase inicial de formação e uma definição de organização da interação, o que influencia as formas da interação consecutivas, como também os resultados obtidos a partir das mesmas. A fase de formação da relação interorganizacional consiste na qualificação da colaboração universidade-empresa, processo seletivo da universidade parceira até a negociação e formalização com a assinatura de documento. Esta primeira fase leva às formas organizacionais, que os autores definem como os arranjos que empresas e universidades estabelecem para operacionalizar o acordo assinado por ambos. A seguir ocorre a fase operacional, propriamente dita, quando as atividades de TC são executadas, gerando os resultados da interação. Ditas atividades são influenciados por fatores facilitadores ou barreiras que intervém positiva ou negativamente no relacionamento entre a universidade e a empresa. Todas as fases supracitadas sofrem a influência de uma motivação para a participação na interação, seja pela universidade, seja pela empresa.

Do ponto de vista empresarial, a literatura dispõe diversas motivações para as empresas se engajarem em atividades de transferência com universidades. São registradas razões desde a busca por alianças baseadas em tecnologia (George, Zahra \& Wood, 2002); o desenvolvimento e a comercialização de novos produtos (Motohashi, 2005); a obtenção de patentes registradas, controle sobre tecnologias prorietárias, ou acesso a resultados de pesquisa (Siegel et al., 2004); a terceirizar de $\mathrm{P} \& \mathrm{D}$ para concluir projetos científicos ou como medida estratégica (Lacetera, 2009); o acesso a conhecimentos mais amplos na busca de vantagem competitiva (Woerter, 2012); o acesso a recursos humanos altamente qualificados e estruturas da universidade (Arvanitis, Sydow e Woerter, 2007); maior velocidade na transferência de tecnologia, especialmente em segmentos de P\&D intensivo (Du et al., 2014); a redução de custos e riscos 
técnicos em P\&D, ou mesmo recursos insuficientes da empresa para P\&D (Caloghirou, Tsakanikas e Vonortas, 2001) o acesso à recursos públicos em projetos de cooperação em P\&D com a universidade (Boehm e Hogan, 2013); a melhoria da imagem e reputação da firma por meio de cooperação com instituições científicas (Lai, 2011); o acesso a estudantes para estágios ou contratação (Ankrah e Al-Tabbaa, 2015); até a melhoria de processos internos e organizacionais (Perkmann, Neely e Walsh, 2011). O consenso na literatura é o de que o processo de interação inicia com a identificação da motivação por parte da empresa.

As atividades de TC nas interações entre universidades e empresas, na perspectiva empresarial, ocorrem em uma diversidade de formatos e natureza. As atividades mais frequentemente descritas na literatura são as comunicações informais e formais entre os membros das universidade e das empresas e o funcionamento de redes de relacionamento (networking) entre as pessoas envolvidas. Dentre as atividades mais formais, encontra-se os contratos de pesquisa conjunta ou exclusiva pela universidade, os contratos de consultoria, e a prestação de serviços por acadêmicos para as empresas, além de treinamentos, ensino e capacitação (Olmos-Penuela, Castro-Martínez, \& D’este, 2014). Por fim, muito relevante é a atividade de licenciamento de novas tecnologias por parte das empresas (Perkmann et al., 2013).

Como fatores facilitadores para as atividades de TC, Bruneel, D'Este e Salter (2010) citam a experiência da empresa em colaborar com universidades, explorando um maior número de canais de interação, com processos internos mais bem estabelecidos para tanto, além da confiança inter-organizacional. Por sua vez, Levy, Roux e Wolf (2009) destacam características de tamanho, foco inovador, proximidade física da universidade e setor de atividade competivivo como fatores que potencializam a interação. De outra parte, são também referidos como fatores que facilita o processo de interação a capacidade de absorção de conhecimento e tecnologia das empresas (Bruneel et al., 2010) como outro, além da qualidade do corpo docente, de pesquisadores e dos órgão intermediários da universidade para a TC, tais como núcleos de inovação tecnológica (NIT) ou agências de TC (Perkmann, King et al., 2011; Lai, 2011).

Já como barreiras, destaca-se a falta de motivação (Sherwood \& Covin, 2008), assim como deficiências de estrutura, processos e comunicação, tanto nas empresas como nas universidades (Hughes \& Kitson, 2013). Além disso, as interações são negativamente influenciadas pela falta, no lado das empresas, de capacidade de absorção ou de retenção do conhecimento e tecnologia (Cohen \& Levinthal, 1990). Citam-se ainda as divergências de finalidade, objetivos institucionais, linguagem e cultura organizacionais entre universidades e 
empresas como obstáculos importantes para as interações entre universidade e empresa (Bruneel; D’Este; Salter, 2010) .

Finalmente, em relação aos resultados esperados pelas empresas das interações com as universidades nas atividades de TC, pontua-se a obtenção de melhor desempenho tecnológico (Leten, Landoni \& Looy, 2014), inovações de produtos, serviços e processos com impacto positivo no nível de competitividade da empresa (Un, Cuervo-Cazurra, Asakawa, 2015); a geração e a adoção de novas tecnologias (Perkmann, King et al., 2011), além do impacto no capital humano e legado de P\&D (Ankrah \& Al-Tabbaa, 2015).

\section{Procedimentos metodológicos}

Este trabalho utilizou a abordagem qualitativa descritiva para analisar as dimensões relevantes no processo de TC entre universidades e empresas. Segundo Gil (1999), as pesquisas exploratórias objetivam desenvolver uma visão geral, aproximada, sobre determinado fato. Estudos exploratórios em profundidade semelhantes foram aplicados por Siegel, Waldman, Atwater e Link (2004), Ankrah et al. (2013) e O'Kane, Mangematin, Geoghegan e Fitzgerald (2015) no contexto de interações de empresas com universidades para TC. Tais estudos utilizaram tal abordagem para o entendimento da perspectiva empresarial sobre fatores antecedentes, intervenientes e consequentes de interações com universidades, o que se assemelha com as características do presente trabalho.

A coleta de dados utilizada foi a de entrevistas de profundidade semiestruturadas com representantes de empresas envolvidas em interações com universidades. Tal técnica é a mesma utilizada em Ankrah et al. (2013) e O’Kane et al. (2015), cujos resultados foram satisfatórios para obter a perspectiva de empresas. O critério inicial para seleção das empresas a serem contatadas foi o reconhecimento como empresas inovadoras. As empresas foram identificadas e as entrevistas prospectadas a partir de listagens de prêmios de inovação do País, tais como, a relação das 100 empresas mais inovadoras do Brasil, do ranking realizado pelo Valor Econômico no de 2015. Outro critério para seleção das empresas convidadas a participar das entrevistas foi o de que tivessem relacionamento com universidades em atividades de TC. Para fortalecer a observação do fenômeno pesquisado, foram priorizadas as empresas que já tivessem produto no mercado decorrente de processos de TC ou histórico relevante de parcerias com a universidade no desenvolvimento de suas iniciativas de inovação. Para tal identificação, foi utilizada também como fonte de nomes de empresas citadas nos censos do Instituto Nacional 
de Propriedade Intelectual (INPI), da Associação Nacional de Pesquisa e Desenvolvimento das Empresas Inovadoras (ANPEI).

$\mathrm{Na}$ seleção dos representantes das empresas, foram consideradas pessoas vinculadas às empresas que tivessem experiência como executivos responsáveis ou de interface com as universidades em processos de TC. O principal critério para seleção dos entrevistados foi a condição de vivência no fenômeno de TC nas interações com universidades. Adicionalmente, foram preferidos os entrevistados com visão estratégica dos assuntos atinentes a inovação e $\mathrm{P} \& \mathrm{D}$ nas interações com universidades.

A Tabela 1 apresenta a listagem das entrevistas realizadas. O levantamento envolveu representantes de 13 empresas com experiência significativa nível de interações com universidades, reconhecidamente inovadoras em nível nacional e internacional - três dessas empresas constam na lista das empresas mais inovadoras do mundo em 2016, segundo a consultoria BCG (The Boston Consulting Group, 2016). Os profissionais entrevistados registraram experiência de pelo menos dez anos no contexto de TC na interação universidadeempresa. Foi considerada ainda no levantamento uma entrevista com representante da Agência de TC de uma universidade Brasileira com larga experiência no tema. As entrevistas foram preponderantemente realizadas por teleconferência e ocorreram no período de Junho a Setembro de 2016. Foram entrevistados profissionais de empresas de diferentes portes, algumas situadas em ambiente universitário (parques tecnológicos ou similares), tanto nacionais quanto estrangeiras. Os entrevistados tinham posições de direção geral, liderança, gerência ou diretoria de áreas de inovação, $\mathrm{P} \& \mathrm{D}$ ou outra área correlacionada com responsabilidade sobre o relacionamento com universidades. 
Tabela 1

Entrevistas Realizadas

\begin{tabular}{|l|l|}
\hline Entrevistado & Posição \\
\hline $\mathbf{1}$ & Direção geral \\
\hline $\mathbf{2}$ & Direção geral \\
\hline $\mathbf{3}$ & Gerência em Inovação, P\&D ou correlata \\
\hline $\mathbf{4}$ & Gerência em Inovação, P\&D ou correlata \\
\hline $\mathbf{5}$ & Diretoria de Agência de Inovação (Universidade) \\
\hline $\mathbf{6}$ & Diretoria em Inovação, P\&D ou correlata \\
\hline $\mathbf{7}$ & Diretoria em Inovação, P\&D ou correlata \\
\hline $\mathbf{8}$ & Diretoria em Inovação, P\&D ou correlata \\
\hline $\mathbf{9}$ & Líder em Inovação, P\&D ou correlata \\
\hline $\mathbf{1 0}$ & Direção geral \\
\hline $\mathbf{1 1}$ & Gerência em Inovação, P\&D ou correlata \\
\hline $\mathbf{1 2}$ & Líder em Inovação, P\&D ou correlata \\
\hline $\mathbf{1 3}$ & Líder em Inovação, P\&D ou correlata \\
\hline $\mathbf{1 4}$ & Diretoria em Inovação, P\&D ou correlata \\
\hline $\mathbf{N a}$ & \\
\hline
\end{tabular}

Nota. O setor de atuação da empresa foi omitido a pedido dos entrevistados.

$\mathrm{Na}$ fase de análise do material coletado, os dados foram primariamente trabalhados utilizando processo de categorização e codificação (Gil, 1999) com recursos do software NVivo (O'Kane, Mangematin, Geoghegan, \& Fitzgerald, 2015). As categorias e subcategorias (dimensões e variáveis) identificadas a partir da revisão da literatura foram utilizadas para a geração do roteiro de entrevistas e também como base conceitual para a comparação com os resultados empíricos obtidos (Yin, 2015). Em essência, seguindo o framework proposto por Ankrah e Al-Tabbaa (2015), foram exploradas as dimensões de motivações, atividades, resultados e intervenientes (facilitadores e barreiras) do processo de TC na interação entre a universidade e empresas. A técnica empregada para esta fase de categorização e codificação foi a análise de conteúdo (Bardin, 2009) evidencia que esta técnica possui dupla função na pesquisa qualitativa: uma função heurística, que contribui para a exploração do conteúdo de forma aberta e que enriquece a obtenção de informações; e a função de administração da prova, por meio da qual as informações obtidas são verificadas de forma sistemática no sentido de sua confirmação ou invalidação. 


\section{Análise dos resultados}

A primeira dimensão analisada é a das motivações das empresas para se engajar em atividades de TC com universidades. Ao serem questionados sobre o que leva as empresas a procurar universidades como fonte de recursos para inovações, os entrevistados elencaram, essencialmente, a busca pelo conhecimento e acesso aos recursos das universidades como as principais motivações.

A busca por conhecimento, seja geral, específico, ou de qualquer outra natureza que ajude na solução de problemas, foi identificada como a principal motivação para interagir com universidades, segundo os representantes das empresas que participaram da pesquisa. Tal constatação está alinhada com a literatura, que indica o acesso ao conhecimento como motivação das empresas (e.g., Ankrah et al., 2013). Nas entrevistas, estão presentes assertivas sobre os motivos para colaborar com universidades colocando primariamente a obtenção do conhecimento, e como consequência da aplicação do conhecimento, a utilização em novos produtos (Hughes \& Kitson, 2013). Nessa linha, o entrevistado 4 descreve as motivações: “[...] são todas áreas novas (de conhecimento), relativamente novas pra gente e que podem gerar novos produtos". Ao descrever a razão de buscar universidades, o entrevistado 12, um executivo da área de inovação, aponta o meio acadêmico como fonte de conhecimento nas áreas de interesse da empresa. “[...] seja em química, seja em materiais, mas a gente vai atrás da referência e do conhecimento mais específico [...] que muitas vezes tá num professor né eh: que é a referência eventualmente pra àquela disciplina no Brasil”".

Um executivo responsável pela área de $\mathrm{P} \& \mathrm{D}$ e inovação da sua empresa acrescentou como motivação o acesso a determinados recursos das universidades. No caso mencionado, a universidade dispõe de vasto banco de microrganismos que ficam disponíveis para testes por parte da empresa de acordo com suas linhas de pesquisa.

A segunda dimensão analisada é a das atividades de TC que as empresas participam em suas interações com as empresas. As atividades identificadas são projetos, produção de conhecimento, cooperação tecnológica, networking ou interação com a rede de relacionamento, prestação de serviços, capacitação, contratação de recursos humanos, fomento à pesquisa e estrutura e pesquisa conjunta. 
A primeira atividade identificada pelos entrevistados é a realização de projetos com as universidades. Na perspectiva do pessoal dessas empresas, estão neste escopo os projetos para a resolução de problemas específicos, pesquisas conjuntas na área de interesse da empresa, ou ainda, trabalhos para acadêmicos dentro das empresas visando dar exposição a situações relevantes para as empresas. Segundo o entrevistado 9, a atividade é assim descrita. “[...] estudantes lá que precisam de casos reais e de entender também um pouco do ambiente da empresa". Observa-se que a empresa denomina esta atividade como uma parceria com a universidade, sem entendê-la como uma relação cliente-fornecedor, que teria apenas um sentido no fluxo de conhecimento. Outro aspecto identificado é a temporalidade dos projetos com as universidades, preponderantemente aqueles com uma orientação de longo prazo.

A produção de conhecimento é mencionada como atividade desenvolvida a partir da interação da universidade com a empresa. Nesta variável encontram-se as atividades de licenciamento, retratadas em Wright, Clarysse, Lockett e Knockaert (2008), Perkmann et al. (2013) e Perkmann, King e Pavelin (2011).

Os entrevistados citam a variável tecnologia como atividade presente nas interações com universidades. Esta atividade é identificada preponderantemente no que tange ao desenvolvimento da tecnologia pela universidade em área de interesse da empresa, além de formas de transferência dessas tecnologias para utilização em novos produtos ou processos empresariais. No contexto desta atividade, os entrevistados fazem referência a agentes como o Fundo de Amparo à Pesquisa no Estado de São Paulo (FAPESP) e a Financiadora de Estudos e Projetos do Ministério da Ciência e Tecnologia (FINEP). Na perspectiva da empresa, o desenvolvimento de tecnologia pela universidade acontece em estágio pré-competitivo. Este ponto é assim descrito pelo entrevistado 13. “[...] ela (a universidade) não gera um produto, ela gera contribuição de um produto". No caso das empresas multinacionais, os entrevistados consideram o desenvolvimento de tecnologia pelas universidades brasileiras como a possibilidade de nacionalização das fontes de inovação empresarial, ao invés de receber todas as tecnologias de fora do País.

Com forte similaridade ao que apontam os trabalhos de Olmos-Penuela, Castro-Martínez e D’Este (2014) e Ankrah e Al-Tabbaa (2015), outra atividade utilizada pelas empresas nas relações com universidades é o networking, ou a interação com a rede de relacionamentos. Esta atividade caracteriza-se pela informalidade e pela diversidade de formas de ocorrer. Os entrevistados citam contatos com professores e alunos de graduação e pós-graduação, eventos em que participam pessoas da empresa junto com acadêmicos, por exemplo, palestras 


\section{Transferência de conhecimento: a perspectiva empresarial}

promovidas pelas empresas que convidam acadêmicos ou pelas universidades que convidam executivos para palestrar. Um executivo, o entrevistado 4, ilustra esta atividade nos seguintes termos. “[...] a gente tem identificado alguns pesquisadores que através de discussões do dia a dia eles disseram: "pô, a gente tem algumas moléculas quem sabe a gente poderia fazer uma parceria".

A primeira dimensão interveniente pesquisada na interação entre universidade e empresa é a das barreiras encontradas para o desenvolvimento de atividades de TC. Como barreiras para a colaboração, as empresas pesquisadas apresentaram os problemas da burocracia, a diferença de foco institucional, dificuldades sobre aspectos negociais e deficiências da universidade. Identifica-se ainda como barreiras para as empresas as expectativas de timing, divergências culturais, falta de comprometimento e falta de integração, assim como, influências negativas do ambiente externo.

Dificuldades oriundas de problemas burocráticos, ou burocracia, é a barreira identificada na formalização dos contratos com as universidades, tanto pela morosidade de resposta quanto pela quantidade de exigências apresentadas no processo. Constata-se que a questão da burocracia é mais evidente nas interações das empresas com universidades públicas. O entrevistado 14 ilustra tal assertiva:

[...] (para) contratar uma fundação, vamos lá, se começa com algumas coisas difíceis, você tem que começar a colocar as coisas naquele programa. Ai pra você firmar o primeiro convênio é muito difícil porque, ã, você começa com a universidade, que passa pela procuradoria da universidade, e aí com procurador federal lá dentro, aí depois que esse cara ver o contrato ele entrega para a fundação, se o jurídico da fundação não concordar com o contrato da universidade, aí começa a confusão.

Outra variação da burocracia na perspectiva das empresas diz respeito ao ambiente regulatório brasileiro, em diferentes setores de atuação. É citado por uma executiva, entrevistado 8, “ “...] na verdade $e$ a gente tem um contexto de pesquisa muito complicado porque apesar do país ser enorme e poder contribuir com pesquisa eles sabem que as coisas não funcionam aqui”. Na literatura internacional, os estudos realizados na perspectiva empresarial não destacam especificamente a barreira burocracia na integração, entretanto, há citações 
específicas em trabalhos como os de Ankrah e Al-Tabbaa (2015) e Bozeman, (2000), que mencionam a burocracia com influência nas interações.

O foco diferente da universidade em relação ao das empresas nas atividades de TC para obter inovação é outra barreira identificada. Com frequência é citado pelos entrevistados o fato de que a mentalidade de recursos humanos da universidade, percebida em suas prioridades, suas motivações, seus interesses, contrasta com a realidade e expectativas do meio corporativo e empresarial. Tal divergência é relatada como um interveniente negativo quando questionados sobre barreiras encontradas nas interações. Assim é ilustrado na entrevista 6:

[...] a universidade tem um hábito de fazer um projeto, publica o trabalho e fecha o circuito, ou seja, eu estou feliz que eu publiquei um trabalho, criei o negócio e não sai nada daquilo, e depois começa outro e outro e outro e então a universidade tem que entender qual é a beleza de fazer um projeto, um trabalho [...].

Outra variável identificada na pesquisa é a negociação que se estabelece entre a empresa e a universidade durante a interação. Tal negociação é colocada como barreira pelas empresas, principalmente sobre questões ligadas a propriedade intelectual e pagamento de royalties. As empresas vêem dificuldades durante o processo negocial, pois percebem que as universidades colocam exigências adicionais no decorrer da negociação, que vão além dos recursos que as empresas estão aportando. Uma executiva entrevistada coloca da seguinte forma na entrevista 11:

[...] ainda dá muito ruído na hora da negociação [...] muitas vezes o que você tem é a universidade mandando orçamentos que incluem itens que não é só porque a empresa é privada que ela pode financiar [...] [não] posso pagar a secretária do departamento entendeu [...] é contrapartida da instituição é um problema da administração pública que não abre concurso, mas não é um problema da empresa.

Outra barreira apontada pelas empresas está relacionada às deficiências da universidade.sz Dentro desta barreira são citados os critérios de avaliação dos pesquisadores, ineficiências organizacionais, falta de conhecimento ou de capacidade técnica em áreas específicas, ou de aplicabilidade dos projetos da universidade e a competição interna no ambiente universitário, assim como a falta de comprometimento por parte dos pesquisadores envolvidos com as empresas. É apontado também a existência de alternativas que as empresas podem contar em relação às universidades, providas por fornecedores mais ágeis. 
As diferentes expectativas de timing, tempos ou prazos de execução, trazem barreiras na interação com as universidades. Executivos citam com frequência que as universidades têm problemas de lentidão na execução e que isso está ligado à própria característica da organização operar, com prazos mais dilatados e sem pressão externa que possa alterar isso. Assim define um executivo sobre esta variável na entrevista 10. “[...] eu acho que o principal ponto é::. é a batida, assim esse [...] as velocidades diferentes e::.... ã::: eles não entenderem como é que funciona o mecanismo de desenvolvimento do produto dentro da universidade". As empresas percebem a divergência, procuram formas de lidar com ela e em alguns casos encontram situações em que a resposta é melhor, como no exemplo de centros de inovação, mencionados a seguir como facilitadores da interação. Entretanto, esta barreira aparece com mais destaque nos resultados desta pesquisa do que no encontrado no trabalho de Cyert e Goodman (1997), que apresentam os problemas de timing dentro de um contexto de outras diferenças de orientação e natureza do trabalho.

Outra dimensão interveniente pesquisada nas atividades de TC entre universidades e empresas é a dos facilitadores. O pessoal entrevistado das empresas traz como facilitadores para interagir com as universidades a existência de agência de interação, o relacionamento entre o pessoal envolvido, a experiência prévia da empresa engajada na interação, os recursos oferecidos pela universidade, a disponibilidade de fomento à pesquisa, os recursos da própria empresa, a cultura e a orientação da universidade e ainda o ambiente externo no entorno das interações.

A variável mais citada como facilitador da interação é a existência de agência na relação universidade-empresa. A pesquisa constata que a agência da universidade por meio de núcleo ou centro de inovação, departamento dedicado e pessoal especializado, contribui, na forma das empresas verem, para com a agilidade nas atividades de TC universidade-empresa. Assim apresenta, na entrevista 7, uma executiva de inovação de empresa que teve mais relações com universidades no passado. "[...] eu tenho visto agora bons movimentos eh: [...] de algumas dessas agências de inovação das universidades, né de [...] elas mesmo tentarem flexibilizar normas internas da universidade pra isso."

Das entrevistas identifica-se o relacionamento sendo outro aspecto atuante como facilitador das atividades de TC entre universidades e empresas. As empresas cultivam networking dentro do meio acadêmico por meio de eventos e contatos informais que promovem 
discussões e colocam na pauta de pesquisadores e professores os assuntos de interesse empresarial. O seguinte trecho da entrevista 4 ilustra este item:

[...] e trouxe uma riqueza de informação que que::: é extremamente valiosa pra busca de novos produtos né [...] e a gente começou a estabelecer essa conexão dentro da própria universidade e isso acabou acelerando a::: a busca ou a::: de soluções né de novos produtos e tudo mais.

Um dos entrevistados, com experiência de longa data no mundo empresarial e acadêmico, destaca que o relacionamento permite a empresa identificar a pessoa certa dentro da universidade, de forma a viabilizar projetos, solucionar problemas e agilizar processos de inovação. Os entrevistados apontam também a qualidade da comunicação, por meio do diálogo entre profissionais das empresas e acadêmicos, como fatores que contribuem para o facilitador do relacionamento. São mencionados casos com influência positiva nas interações em que o executivo ou especialista da empresa já passou pelos quadros de universidade, ou vice-versa, e isso permite que os interlocutores "falem a mesma língua". Na visão das empresas, contribui também para o relacionamento a proximidade com as instalações físicas da universidade, pois permite que situações do dia-a-dia sejam resolvidas com mais praticidade e em menos tempo. Sobre o aspecto da proximidade geográfica, já constatavam Levy, Roux e Wolf (2009), Abramo, D’angelo, Di Costa e Solazzi (2011), Leten, Landoni e Looy (2014), Maietta (2015) e Ankrah e Al-Tabbaa (2015), sobre como isso ajuda no intercâmbio de conhecimento. Dentro do tema relacionamento, mais especificamente no que tange aos níveis de confiança interorganizacional, encontra-se base nos estudos de Bruneel, D'este e Salter (2010), Perkmann, Neely et al. (2011) e Ankrah e Al-Tabbaa (2015).

Boa parcela dos executivos entrevistados referem a experiência da própria empresa ou de seus colaboradores em interações com universidades como variável de facilitação às atividades de TC. A experiência é referida como facilitador de uma maneira geral, pois o pessoal das empresas utiliza as "lições aprendidas" no relacionamento com as universidades para melhorar os resultados. Em outras instâncias, as empresas citam especificamente as vantagens de ter aprendido com situações semelhantes para negociar contratos, desenvolver projetos, tratar questões de licenças, entre outras. A experiência específica em TC com universidades é citada pelas empresas como facilitador, pois a empresa passa a ter uma perspectiva estratégica para lidar com a parceria acadêmica. Segue o depoimento do entrevistado 10 acerca deste ponto: 
[...] o que eu aprendi nesses anos é que tu não pode(s) chegar na universidade com necessidades imediatistas, tem que chegar com projetos de médio e longo prazo [...] projetos de no mínimo mais de vinte e quatro meses $\tilde{a}:::$ [...] no mínimo do mínimo seria dezoito meses, vinte e quatro meses, chegar com uma coisa de seis meses um ano.

O conjunto dos recursos da universidade a serviço da colaboração com a empresa também figura como elemento facilitador do processo de TC. Executivo de uma multinacional atuante no Brasil aponta que o nível de eficiência da universidade facilita a colaboração. Ele descreve tal eficiência como aparente principalmente no "nível de organização da instituição", na fluência das informações com impacto na qualidade da comunicação universidade-empresa e na agilidade do processo decisório da universidade. Outros entrevistados trazem como recursos facilitadores para interação os recursos humanos (competência dos acadêmicos), financeiros, materiais (infraestrutura, equipamentos) e de informações por meio de bancos de dados softwares mantidos e desenvolvidos pelas universidades. Na entrevista 1, o seguinte trecho é ilustrativo sobre este fator. "[...] a mesma vantagem que eu estou procurando na universidade: recursos humanos [...] método, informação, a universidade é ótima nisso. Se tem as melhores cabeças, estão, nas universidades". Ademais, aponta-se a experiência da universidade nas interações com as empresas, pois a instituição desenvolve recursos que facilitam o relacionamento e melhoram os resultados das interações. Nos trabalhos recentes de Perkmann, King et al. (2011) e Boehm e Hogan (2013), a qualidade dos docentes e dos colaboradores acadêmicos é apontada como facilitador na perspectiva das empresas que interagem com universidades. Desta forma, observa-se um alinhamento parcial do que se encontra na literatura com os achados desta pesquisa no que diz respeito a este facilitador.

Outro interveniente facilitador identificado é o fomento à pesquisa por meio de financiamentos de projetos de pesquisa para fins de inovação. As entrevistas apontam interveniência positiva pela maior disponibilidade de recursos públicos, no âmbito estadual ou federal, para aplicação em projetos com participação de universidades. O entrevistado 6 descreve da seguinte forma:

[...] o governo brasileiro com as universidades tem que fazer o seu papel de criar uma cadeia produtiva forte mas tem que ter incentivo estadual e federal pra que 
seja viável empresas e universidades trabalharem [...] porque se não existir isso se torna inviável, né, é impossível.

As empresas têm a percepção de que universidade que tem mais acesso a fontes de financiamento à pesquisa e que faz uso de tal recurso, tem melhores condições de responder às demandas das atividades de TC visando inovações empresariais. Por outro lado, aquela universidade que vê a empresa meramente como uma financiadora de seus projetos acadêmicos, apresenta mais limitações como fonte de inovação oriundas das interações entre elas. Na visão dos entrevistados, encontra--se mais efetividade na atuação de empresas e universidades como parceiras em projetos de inovação financiados por recursos para fomento à pesquisa, tais como FINEP e FAPESP.

A última dimensão analisada refere-se aos resultados esperados pelas empresas que têm nas interações com universidades fontes de inovação. A variável de maior incidência foi a do desenvolvimento de novos produtos oriundos da interação universidade-empresa. Também foram identificadas as variáveis de melhorias em processos e de imagem empresarial, assim como, resultados mercadológicos.

A maior parte dos executivos que participaram da pesquisa apresentam como resultado esperado o desenvolvimento de novos produtos a partir da interação de suas empresas com universidades. Segundo os entrevistados, as empresas esperam que as universidades tragam conhecimento em novos elementos que sejam insumos para novas tecnologias a serem comercializadas no mercado. No setor de uma das empresas da lista de entrevistas realizadas, a descoberta e a disponibilidade de novas moléculas é resultado que a empresa obtém ao interagir com o meio acadêmico. O foco da empresa na interação com a universidade é que novos produtos sejam desenvolvidos e introduzidos no mercado. O entrevistado 4 descreve da seguinte forma este resultado. "[...] são todas áreas novas, relativamente novas pra gente e que podem gerar novos produtos". Outro aspecto identificado, é que as universidades participam mais ativamente em estágios anteriores no desenvolvimento de conhecimento científico em novas tecnologias que serão aplicadas em novidades futuras no mercado. Assim apresenta o entrevistado 10. “[...] então eu espero conseguir enxergar mais coisas na universidade onde eu possa buscar tecnologias e simplesmente agregar nos meus produtos".

A melhoria em processos empresariais, sejam eles internos ou ao longo da cadeia de valor da empresa, é outro resultado identificado. Executiva de empresa multinacional cita a inovação em processos que geram mais produtividade e assim aumenta a competitividade da 
empresa, e de forma agregada, do país como um todo. Assim, a mudança oriunda da TC entre universidade-empresa melhora os resultados dos processos empresariais pela utilização de certo know-how desenvolvido pela universidade e transferido para a empresa. Os processos citados pelas empresas estão em diversos contextos empresariais, são citados como exemplo resultados de melhorias em processos logísticos, especificamente em seus aspectos regulatórios, tanto nas questões do meio ambiente, quanto tributárias. Refere-se também como resultados esperados pela empresa a utilização de programa de computador desenvolvido dentro da interação da empresa com universidades. Nos trabalhos de George, Zahra e Wood (2002), Perkmann, Neely et al. (2011), Ankrah et al. (2013) e Un e Asakawa (2015) encontram-se preceitos teóricoconceituais sobre inovações em processos de empresas que obtiveram isso da interação com o meio acadêmico, bem como, do aumento de competitividade advindos de tal colaboração.

\section{Discussão dos resultados}

Os resultados encontrados na pesquisa permitem a construção de um novo desenho conceitual para melhorar o entendimento sobre os fatores importantes para as empresas que se engajam em atividades de TC com universidades. Este desenho é apresentado na Figura 2 e é discutido a seguir.

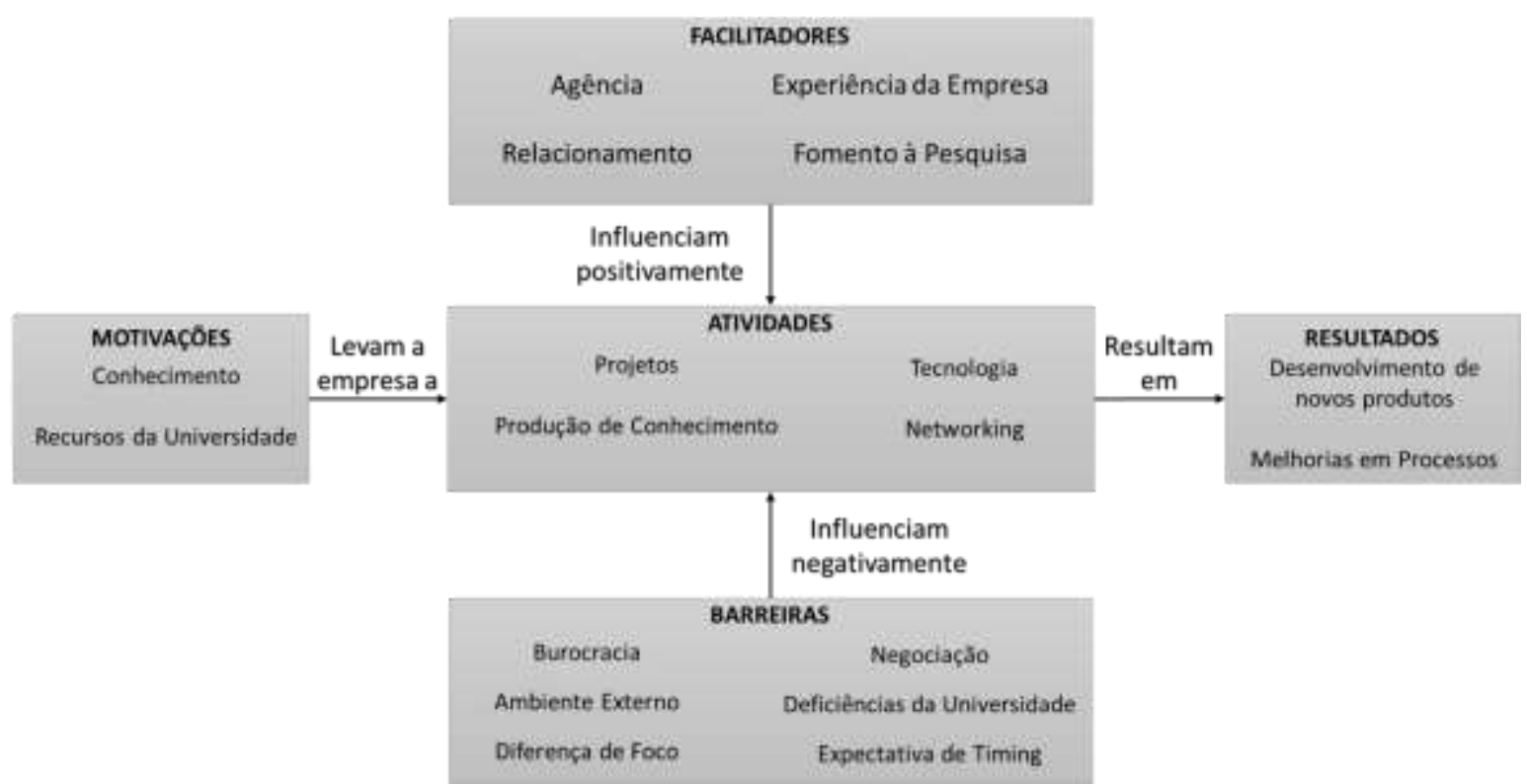

Figura 2. Processo conceitual das empresas envolvidas em TC com universidade

As empresas buscam interagir com as universidades para obter conhecimento e acessar recursos que as universidades oferecem, contribuindo para as inovações empresariais. As 
atividades de TC ocorrem de várias formas. De um lado, as atividades se configuram em interações estruturadas e institucionais, tais como, projetos conjuntos, contratos de prestação de serviços, treinamentos ou a utilização conjunta de estrutura para fins de pesquisa. Porém, as colaborações universidade-empresa também ocorrem informalmente e de forma mais individualizada, como se caracteriza nas redes de relacionamento entre profissionais das empresas e meio acadêmico, contratação de pessoas com histórico acadêmico pelas empresas, produção conjunta de trabalhos científicos, entre outros. Observa-se nesta dimensão da interação interorganizacional, que há maior intensidade e efetividade nas atividades em TC quando as empresas são protagonistas das ações de interação, aproveitando-se da proximidade física ou de seu pessoal qualificado com background acadêmico.

Dentre os fatores intervenientes estão as dimensões como polos opostos de influência das barreiras e dos facilitadores. Na influência negativa, que impacta na execução e operação das colaborações, encontram-se diversas barreiras. Dentre elas estão, preponderantemente, variáveis internas encontradas nas universidades, tais como, a burocracia, a divergência do foco de atuação, a percepção das empresas de falta de comprometimento dos parceiros acadêmicos por questões culturais, deficiências funcionais ou estruturais, ou ainda diferenças na noção de prazos para atendimento das demandas de inovação. Outras barreiras estão ligadas a interação interinstitucional, como as negociações de direitos e obrigações ou a falta de integração entre as equipes envolvidas. Por fim, aparecem também barreiras oriundas das condições ambientais, em especial nos aspectos legais e político-institucionais.

Os fatores intervenientes facilitadores aparecem como soluções para as dificuldades trazidas pelas barreiras. A existência de intermediários para as interações universidade-empresa configura-se como resposta a diversas divergências institucionais. A experiência da empresa e seus recursos, além do relacionamento com pessoal das universidades, são outras variáveis de facilitação para as atividades com impacto positivo nos resultados das interações. O ambiente natural e recursos encontrados nas universidades são também facilitadores observados.

Os resultados esperados representam desempenho em termos de inovações que são obtidos pelas empresas que interagem com as universidades. Como resultado esperado pelas empresas, é citado o desenvolvimento de novos produtos para o mercado. Em alguns casos, espera-se obter como resultado o aperfeiçoamento de processos empresariais internos com tecnologia obtida junto ao meio acadêmico. Também aparecem aspectos de melhorias de imagem e de posição de mercado como resultados das atividades de TC com universidades. 


\section{Considerações finais}

Este estudo apresenta como conclusão uma perspectiva empresarial sobre as interações entre empresas e universidades na realidade brasileira. As empresas têm as universidades como fontes de inovação e as atividades de TC consistem no mecanismo que transforma a interação entre universidade e empresa em resultados empresariais. Assim, justifica-se a importância de estudar as atividades de TC, na análise dos seus fatores antecedentes, intervenientes, as formas de interação e benefícios efetivamente obtidos pelas empresas.

A primeira dimensão observada - das motivações das empresas que se engajam em TC com a universidade, permitiu a identificação de dois fatores antecedentes por parte das empresas: conhecimento (do meio acadêmico) e recursos da universidade. As principais motivações observadas são a busca pelo conhecimento e acesso aos recursos das universidades.

A segunda dimensão mapeada, das atividades de TC nas relações entre universidade e empresas, indicou como as principais atividades, projetos, produção de conhecimento, cooperação tecnológica e networking ou interação com a rede de relacionamento.

No que tange às barreiras e os facilitadores na percepção das empresas quando das suas interações com universidades em atividades de TC, foram analisadas separadamente as duas dimensões intervenientes. As principais barreiras evidenciadas são os problemas da burocracia, a diferença de foco institucional, dificuldades sobre aspectos negociais e deficiências da universidade. Quanto aos facilitadores, observa-se os principais como sendo a existência de agência de interação, o relacionamento entre o pessoal envolvido, a experiência prévia da empresa engajada na interação, os recursos oferecidos pela universidade, a disponibilidade de fomento à pesquisa, os recursos da própria empresa, a cultura e a orientação da universidade e ainda o ambiente externo no entorno das interações.

A quarta e última dimensão - dos resultados que as empresas esperam a partir da interação com universidades em atividades de TC, apresentou o desenvolvimento de novos produtos e a melhoria em processos pela utilização de novas tecnologias, como as principais variáveis observadas.

Com a ideia de que as empresas são as protagonistas na inovação oriunda das interações com universidades, as variáveis identificadas neste estudo podem servir como base para ações mais efetivas nesta forma de relação institucional. 


\section{Referências}

Abramo, G., D'angelo, C, Di Costa, F., \& Solazzi, M. (2011). The role of information asymmetry in the market for university-industry research collaboration. Journal of Technology Transfer, 36, 84-100

Ankrah, S. N., Al-Tabbaa, O. (2015). Universities-industry collaboration: a systematic review. Scandinavian Journal of Management, 31(3), 387-408

Ankrah, S. N., Burguess, T. F., Grimshaw, P., \& Shaw, N. E. (2013). Asking both university and industry actors about their engagement in knowledge transfer: what single-group studies of motives omit. Technovation , 33, 50-65

Arvanitis, S., Kubli, U., Sydow, N., \& Woerter, M. (2007). Knowledge and Technology Transfer (KTT) activities betweenuniversities and firms in Switzerland - the main facts: an empirical analysis based on firm-level data. The ICFAI Journal of Knowledge, 5,(6)

Bardin, L. (2009). Análise de conteúdo (4a ed.). Lisboa: Setenta

Belderbos, R., Carree, M., \& Lokshin, B. (2004) Cooperative R\&D and firm performance. Research Policy, 33, 1477-1492

Boehm, D. N., \& Hogan, T. (2013). Science-to-Business collaborations: a science-tobusiness marketing perspective on scientific knowledge commercialization. Industrial Marketing Management, 42, 564-579

Bozeman, B. (2000). Technology transfer and public policy: a review of research and theory. Research Policy, 29, 627-655

Bruneel, J., D'este, P., \& Salter, A. (2010). Investigating the factors that diminish the barriers to university-industry collaboration. Research Policy 39, 858-868

Caloghirou, Y., Tsakanikas, A., \& Vonortas, N. S. (2001). University-industry cooperation in the contexto of the european framework programmes. Journal of Technology Transfer 26, pp. 153-161

Closs, L., \& Ferreira, G. C. (2012). A transferência de tecnologia universidade-empresa no contexto brasileiro: uma revisão de estudos científicos publicados entre os anos 2005 e 2009. Gestão \& Produção, 19, (2), 419-432

Cohen, W. M., \& Levinthal, D. A. (1990). Absorptive capacity: a new perspective on learning and innovation. Administrative Science, 35, (1), 128-152

Cyert, R. M., \& Goodman, P. S. (1997, spring). Creating effective university-industry alliances: an organizational learning perspective. Organizational Dynamics, 45-57

de Wit-de Vries, E., Dolfsma, W. A., van der Windt, H. J., \& Gerkema, M. P. (2018). Knowledge transfer in university-industry research partnerships: a review. The Journal of Technology Transfer, 1-20. Publicado online. https://doi.org/10.1007/s1096.

Frank, A. G., Cortimiglia, M. N., Ribeiro, J. L. D., \& Oliveira, L. S. (2016). The effect of innovation activities on innovation outputs in the Brazilian industry: Market-orientation vs. technology-acquisition strategies. Research Policy 45, (3): 577-592.

Freitas, I. M. B., Marques, R. A., \& Silva, E. M. P. (2013). University-industry collaboration and innovation in emergent and mature industries in new industrialized countries. Research Policy, 42, 443-453

George, G., Zahra, S. A., \& Wood, D. R. (2002). The effects of business-university alliances on innovative output and financial performance: a study of publicly traded biotechnology companies. Journal of Business Venturing, 17, 577-609

Gil, A. C. (1999). Métodos e técnicas de pesquisa social (5a ed.). São Paulo: Atlas 
Hughes, A., \& Kitson, M. (2013). Connecting the Ivory Tower: business perspectives on knowledge exchange in the UK. UK Innovation Research Center, Cambridge

Kahn, K. B. (2018). Understanding innovation. Business Horizons, 61(3), 453-460.

Lai, W. H. (2011). Willingness-to-engage in technology transfer in industry-university collaborations. Journal of Business Research, 64, 1218-1223

Leten, B., Landoni, P., \& Looy, B. V. (2014). Science or graduates: how do firms benefit from the proximity of universities ? [Versão eletrônica]. Research Policy, 43(8), 13981412. Recuperado de <http://doi.org/10.1016/j.respol.2014.03.005>

Levy, R., Roux, Æ. P., \& Wolff, Æ. S. (2009, fevereiro). An analysis of science - industry collaborative patterns in a large European University. Journal of Technology Transfer, 34(1), 1-23. Recuperado de <http://doi.org/10.1007/s10961-007-9044-0>

Maietta, O. W. (2015). Determinants of university-firm R \& D collaboration and its impact on innovation: a perspective from a low-tech industry. Research Policy, 44, 1341-1359

Motohashi, K. (2005). University-industry collaborations in Japan: the role of new technology-based firms in transforming the National Innovation System. Research Policy, 34, 583-594

O'Kane, C., Mangematin, V., Geoghegan, W., \& Fitzgerald C. (2015). University technology transfer offices: the search for identity to build legitimacy. Research Policy, 44(1), 421-437

Olmos-Penuela, J., Castro-Martínez, E., D’este, P. (2014). Knowledge transfer activities in social sciences and humanities: explaining the interactions of research groups with nonacademic agents. Research Policy, 43, 696-706. Recuperado de $<$ http://doi.org/10.1016/j.respol.2013.12.004>

Perkmann, M., King, Z., \& Pavelin, S. (2011). Engaging excellence? Effects of faculty quality on university engagement with industry. Research Policy, 40, 539-552

Perkmann, M., Neely, A., \& Walsh, K. (2011). How should firms evaluate success in university-industry alliances? A performance measurement system. $R \& D$ Management, 41, 202-216

Perkmann, M., Tartari, V., McKelvey, M., Autio, E., Broström, A., D’Este, P., Fini, R., Geuna, A., Grimaldi, R., Hughes, A., Krabel, S., Kitson, M., Llerena, P., Lissoni, F., Salter A., \& Sobrero, M. (2013). Academic engagement and commercialisation: a review of the literature on university-industry relations. Research Policy, 42(2), 423-442

Ryan, P., Geoghegan, W., Hilliard, R. (2018). The microfoundations of firms' explorative innovation capabilities within the triple helix framework. Technovation, 1-13. Publicado online. https://doi.org/10.1016/j.technovation.2018.02.016.

Sherwood, A. L., \& Covin, J. G. (2008). Knowledge acquisition in University-Industry alliances: an empirical investigation from a learning theory perspective. The Journal of Product Innovation Management, 25, 162-179

Siegel, D. S, Waldman, D. A., Atwater, L. E., \& Link, A. N. (2004). Toward a model of the effective transfer of scientific knowledge from academicians to practitioners: qualitative evidence from the commercialization of university technologies. Journal of Engineering and Technology Management, 21(1-2), 115-142

Un, C. A., Cuervo-Cazurra, A., \& Asakawa, K. (2015). Types of R \& D Collaborations and Process Innovation: The Benefit of Collaborating Upstream in the Knowledge Chain. Journal of Product Innovation Management, 32, 138-153

Woerter, M. (2012). Technology proximity between firms and universities and technology transfer. Journal of Technology Transfer, 37, 828-866). Recuperado de <http://doi.org/10.1007/s10961-011-9207-x> 
Wright, M., Clarysse, B., Lockett, A., \& Knockaert, M. (2008). Mid-range universities linkages with industry: knowledge types and the role of intermediaries. Research Policy, 37, 1205-1223. Recuperado de <http://doi.org/10.1016/j.respol.2008.04.021>

Yin, R. K. (2015). Estudo de caso: planejamento e métodos (5a ed.). Porto Alegre: Bookman 